مجلة جامعة الدلك عبدالعزيز: علوم البحار، م29 ع1، 91 - 103 صفحة (1440هـ / 2019م)

DOI :10.4197/Mar.29-1.6

\title{
محاكاة ظاهرة التوغل الملحي في نهر شط العرب - جنوب العراق
}

\section{سامر عدنان الطائي، وصادق سالم عبداللها، وإياد عبد الجليل المهري2}

1"قسم فيزياء المصبات والمياه البحرية، مركز علوم البحار، وقسم الجغرفيا، كلية التربية للبنات، جامعة البصرة، بصرة، العراق

Samer.adnan74@gmail.com

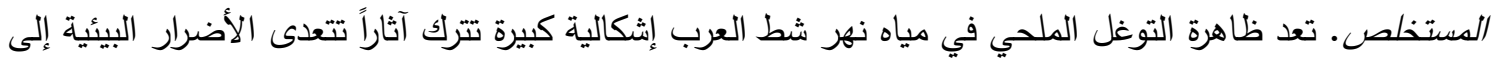
الواقع الاقتصادي والاجتماعي على مدينة البصرة انطلاقا من كون هذا النهر شريان هذه المدينة ورئتها المائية،

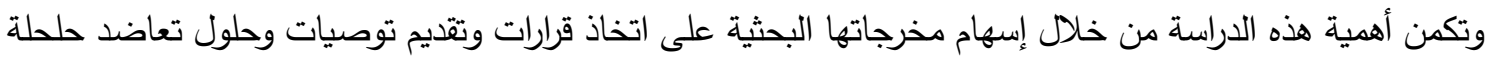

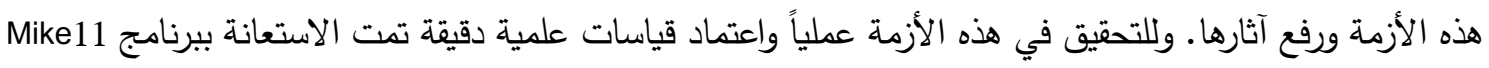

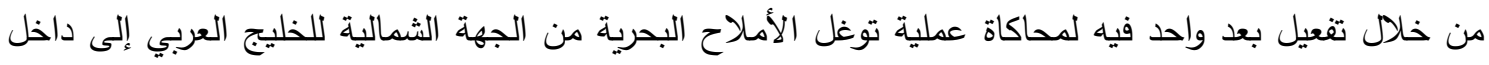

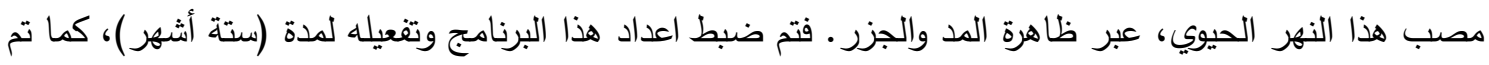

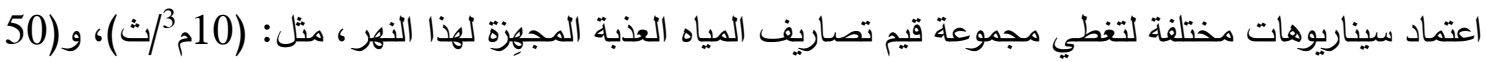

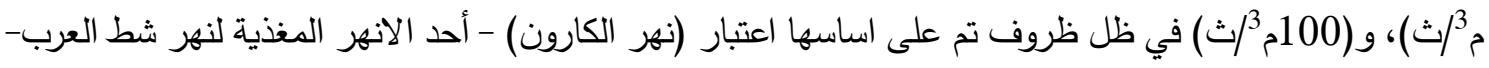

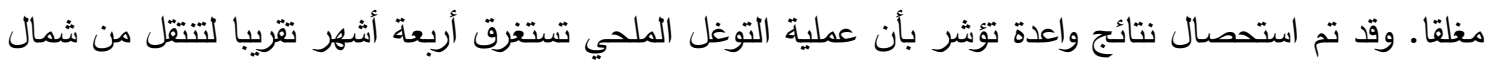

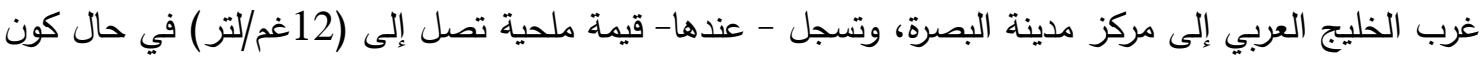

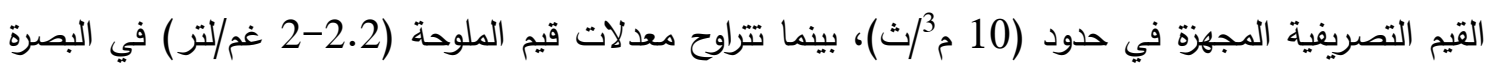

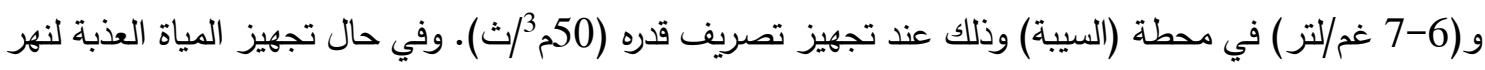

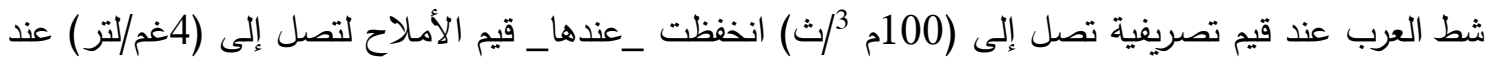

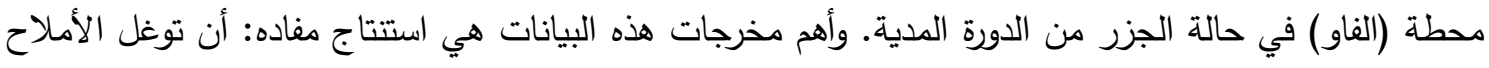

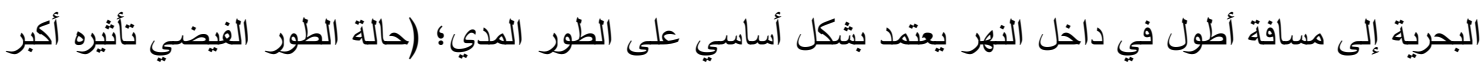
من حالة الطور المحاقي) وتجهيز النهر بالمياه العذبة. الكلمات الدفتاحية: توغل الأملاح، المحاكاة، mike11، تصريف المياه العذبة، مصب نهر شط العرب. 
لهي أكثر تأثراً بكميات تصاريف المياة العذبة، كما ان ديناميكية التوغل فيها تكون عبر (ظاهرة الانتشار) بخلاف ما هو عليه الحال ك(ظاهرة انتقال موجة مدية) . [Leblond, 1978; Gong and Shen, 2011; Al-Taei et al., 2014] من هنا جاءت هذه الدراسة لتضع تصورًا خاصًا حول عملية توغل الأملاح خلال مياه شط العرب على شكل موديل رياضي يمكن التتبؤ من خلاله بمعرفة الفترة الزمنية التي تحتاجها الأملاح ومقدار قيمها للوصول إلى مركز مدينة البصرة.

\section{1-1 منطقة (الدراستة}

يتكون نهر شط العرب من التقاء نهري دجلة والقرات عند مدينة القرنة ويجري بعد ذلك بالاتجاه الجنوبي الثرقي ولمسافة (204 كم) لتصب مياهه في شمال الخليج العربي جنوب مدينة الفاو، ليكوّن له مصباً يدعى (مصب شط العرب) كما في الشكل 1. يعد نهر شط العرب من الانهر المدية [عبد الله، 1990؛؛ وذلك لتأثره بظاهرة المد والجزر القادمة من الخليج العربي. كما ان نوع المد والجزر فيه هو من النوع المختلط - السائد نصف اليومي [Abdullah, 2002]. يتموضع شط العرب جغرافيا في منطقة تتميز بمناخ شبه جاف يتصف بارتفاع درجات الحرارة صيفاً وانخفاضها شتاءً، ما جعله يتسم بشحة الامطار وارتفاع معدلات التبخر ، وهذه الظروف تتسحب بشكل جلي على هيدرولوجية النهر [المحمود، 2011] الذي يبلغ معدل عرضها ( 400 متر) وتتراوح اعماقه بين (8 - 15متر).

\section{1. المقدمة}

تتعرض الأنهار المرتبطة بالبحار إلى تأثير طاقتين؛ أولهما: تصريف المياه العذبة المتدفقة من اليابسة، والثانيهما: ظاهرة المد والجزر القادمة من البحر. وتتبادل الأدوار بينهما وفقا لقيمة تصريف المياه العذبة؛ اذ تتدفع المياه العذبة باتجاه البحر عندما تكون كمية التصريف كبيرة بينما يحدث العكس فتتوغل الأملاح عندما تتخفض كمية التصريف مسببة تغييرا في نوعية مياهه، لاسيما في منطقة المصب؛ كون هذه المنطقة أقرب لتأثير المياه البحرية أكثر من تأثير Cai et [تصريف المياه العذبة القادمة من أعلى النهر .[Al-Taei et al., 2014; al., 2015 ولأهمية مناطق المصبات أقتصادياً وصناعياً وبيئياً في العالم يعد موضوع توغل الأملاح ظاهرة علمية كونه أكثر الموضوعات اشتغالا وبحثاً من لدن المنظمات والمراكز البحثية. فالخصائص الهيدروديناميكية للأنهار المدية ناتجة من التداخل بين عمليات تصريف مياه النهر من منابعها العذبة وظاهرة المد البحري المالح، حيث ينتج عنه بعض الإشكاليات المؤثرة مثيل: الفيضانات أو توغل الأملاح ل Savenije, 2008; Vongvisessomjai and .[Catanantavet, 2006

فظاهرة توغل الأملاح القادمة من البحار باتجاه الأنهار العذبة المتصلة بها على نحو مباشر عبر ما يدعى بـ (المصبات) من خلال ظاهرة الانتقال الطولي 


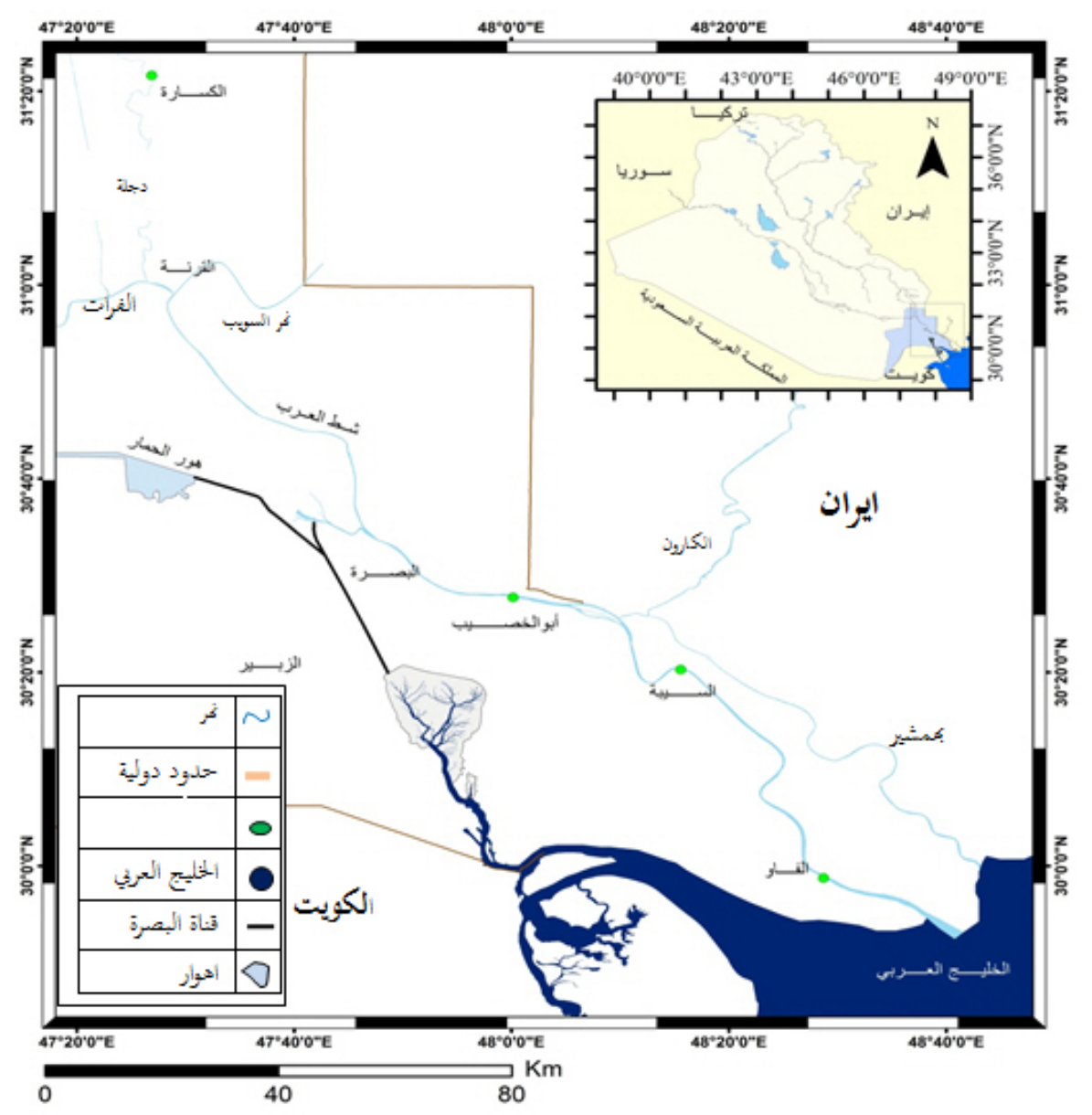

شكل 1. منطقة الاراسة.

فتتأثر هيدروديناميكية جريان المياه في نهر شط عند مدينة الفاو (2.1 متر ) تقربباً [مركز علوم البحار، العرب بطاقتي المد والجزر المتعاكستين، تتمثل الأولى 2014] وتتبادل الطاقتان المتدافعتان الادوار وفقاً لقوة ومقدار كل طاقة على حساب الأخرى، لكن تاريخياً بالطاقة التصريفية الدافعة للمياه العذبة القادمة من وخلال العقود الماضية كانت الهيمنة لصالح طاقة روافد النهر العليا (دجلة، الفرات) حيث تقدر كمية التصريف فكان لها الدور الأساس في تحرك عجلة التصريف عندها بأكثر من (1000م3/ث) في ستينات المياه لصالحها وخلف ذلك مخرجات تتناسب وطبيعة القرن الماضي لتصل في الوقت الحاضر إلى هذه القوة من فيضانات وترسيبات ونمو دلتا مصب (50م3/ث) فقط [وزارة الموارد المائية، 2016]، اما شط العرب. إلا أن الوقت الحالي شهد تبدلا في الادوار الطاقة الأخرى فهي طاقة المد البحري المعاكسة والقادمة من أسفل النهر حيث يبلغ مدى المد والجزر لصالح قوة التدافع البحري القادمة من الأسفل بتأثر 
قبل جهة دانماركية مانحة (DANIDA) إلى مركز علوم البحار-جامعة البصرة، ويككن من خلاله الحصول على صيغ تحاكي سرعة التيارات ونوعية المياه ونقل الرواسب في الأنهار والمصبات وغيرها

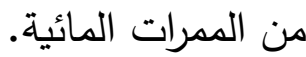

يعتمد النموذج ذو البعد الواحد 1D على

معادلات سانت-فينانت (Saint-Venant) التفاضلية غير الخطية المعتمة على الزمن والمعرفة بمعادلات الاستمرارية والحركة والانتشار وعلى النحو التالي:

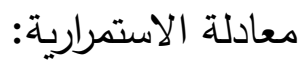

$$
\begin{gathered}
\frac{\partial Q}{\partial t}+\frac{\partial A}{\partial t}=q \\
\alpha \frac{\partial q}{\partial t}+\frac{\partial}{\partial x}\left(\beta \frac{\mathrm{Q}^{2}}{A}\right)+g A \frac{\partial h}{\partial x}+\frac{Q|Q|}{\mathrm{C}^{2} \mathrm{RA}}=0 \\
\frac{\partial \mathrm{AAC}}{\partial \mathrm{t}}+\frac{\partial \mathrm{QC}}{\partial \mathrm{x}}-\frac{\partial}{\partial \mathrm{x}}\left(\mathrm{AD} \frac{\partial \mathrm{C}}{\partial \mathrm{x}}\right)=-\mathrm{AKC}+\mathrm{C}_{2} \mathrm{q} \text { الانتشار } \mathrm{d} \text { (1) }
\end{gathered}
$$

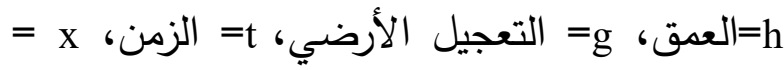
اتجاه الجريان، Q= التصريف، R= نصف القطر الهيدروليكي، C= عامل شيزي، $\beta$ ،ر= دوال تعتمد على التصريف، K= الاستيعاب conveyance. والنموذج أعلاه يقوم بايجاد صيخ توزيع مناسيب المياه والسرعة والتصريف وفقاً للوقت في المقطع

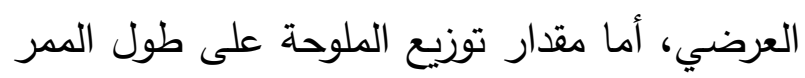

انخفاض قوة التصريف ما أدى إلى تغلب طاقة المد، ونتج عن ذلك حدوث إثكالايات ملازمة لقوة المد تتمثل في ازدياد طاقة الأمواج عند راس المصب في في لمدي مدخل النهر عند مدينة الفاو، وما يرافقها من عمليات تعرية في دلتا النهر هذا بالإضافة إلى التأثير السلبي على نوعية المياه بسبب توغل اللسان الملحي الزاحف

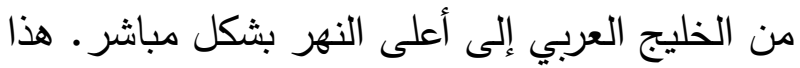
وتعد الدراسات الخاصة بلى لبوضوع المحاكاة الهيدرودينامكية لنهر شط العرب وفقا للتأثير المتبادل بين التصريف والتأثير المدي قليلا جدا قياسا بحجم هذه الاشكالية المتعاظمة حيث قدمت شركة [لين [et al., 1981 البولونية دراسة بينت فيها ان توغل فناس الأملاح من الخليج العربي يصل إلى مسافة (30 كم) أعلى النهر بوجود تصريف مياه عذبة من الأعلى يبلغ مقدار (100 م³/ث). بينما قدم ائتلاف شركات إيطالية [CSED et al., 2012] دراسة أقر فيها بوجود

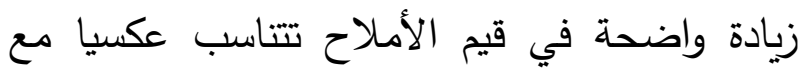
انخفاض قيم تصاريف المياه العذبة، ولكن لم تتفق لتصادئ الدراستان بمصدرية الأملاح، هل هو الخليج العربي أو مصادر أخرى، ولكن ارتفاع تراكيز ملوحة مياه النهر يعود إلى مصادر متتوعة.

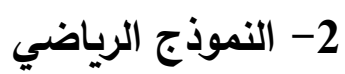

هike11

من قبل معهد الهايدرولكس الدنيماركي ( Danish (Hydraulic institute (DHI) 
2- استخدام برنامج المحاكاة Mike11 ذو

البعد الواحد والدصمم من قبل معهر (الهيدرولكس)

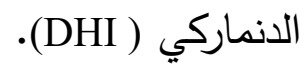

3- استخدمت بيانات المد والجزر (الارتفاعات والانخفاضات) للفترة من 1-1-2015 وإلى 31-12-2017 أعتمادا من جداول المد والجزر الصادرة من الثركة العامة لموانئ العراق.

استخخلت - أيضا- صورة جوية لمنطقة الدراسة إلى برنامج Mike11 لتكون أكثر موائمة للحالة الواقعية عندما اكتسبت الوضع الجغرافي لها وذلك وفقاً للإحداثيات الجغرافية وتكون لها صورة على شكل شبكة (gird) كما في شكل 2، إذ تم تمثيل النهر

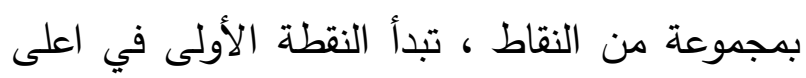

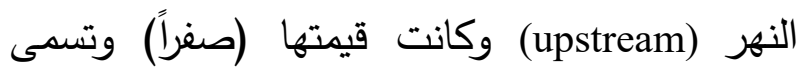
ضمن البرنامج (chainage 0.0) وهكذا يمكن استحصال بعد النقاط التي تليها من خلال موقع هذه النقطة، ومعرفة عدد وبعد النقاط التي تم ادراجها وفقاً لطول النهر وخلال ذلك يمكن تغطيته بالثكل الملائم للدحاكاة، إضافة إلى ذلك تم استخدام الطريقة ذاتها

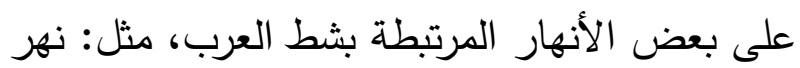

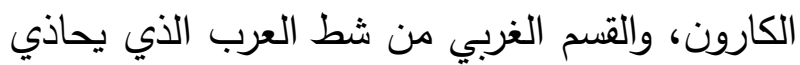
جزيرة (أم الرصاص) وجزء من (نهر الكرمة) لتكون

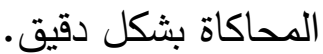

المائي فيمكن إيجادها بعد معرفة المتغيرات الهيدروليكية وتوزيعها [DHI, 2007].

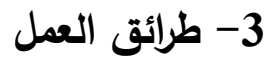

العمل الميداني: تضمنت القياسات الحقلية قياس مقدار التصريف، وسرعة التيارات واتجاهها في عدة محطات هي: (البصرة، والسيبة، والفاو) كما في شكل 1، موزعة على طول النهر، حيث تمثل كل محطة شكل مقطع عرضي، كما تم قياس مقدار قيم

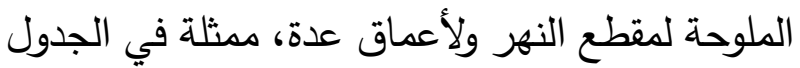
أدناه. واستخدم لقياس التصاريف جهاز نوع (ADCP Acoustic Doppler Current Profiler,Teledyne, Co. USA ((Senso Direct 150) نوع Lovibond Multimeter) بثكل مباشر بالعمل الميداني وجمعت عينات خلال الدورة المدية والبالغة (25ساعة) وأجري فحصها في بعلي مختبرات مركز علوم البحار/جامعة البصرة بجهاز Digital Salinometer E-202, ,OGAWA SEIKI) (Co. LTD.,Tokyo, Japan

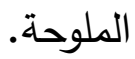

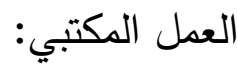

1- استخدمت البيانات الخاصة بالمح الطوبوغرافي والباثيمتري لنهر شط العرب من قبل

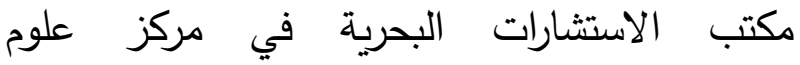
البحار/جامعة البصرة خلال السنوات 1998- 


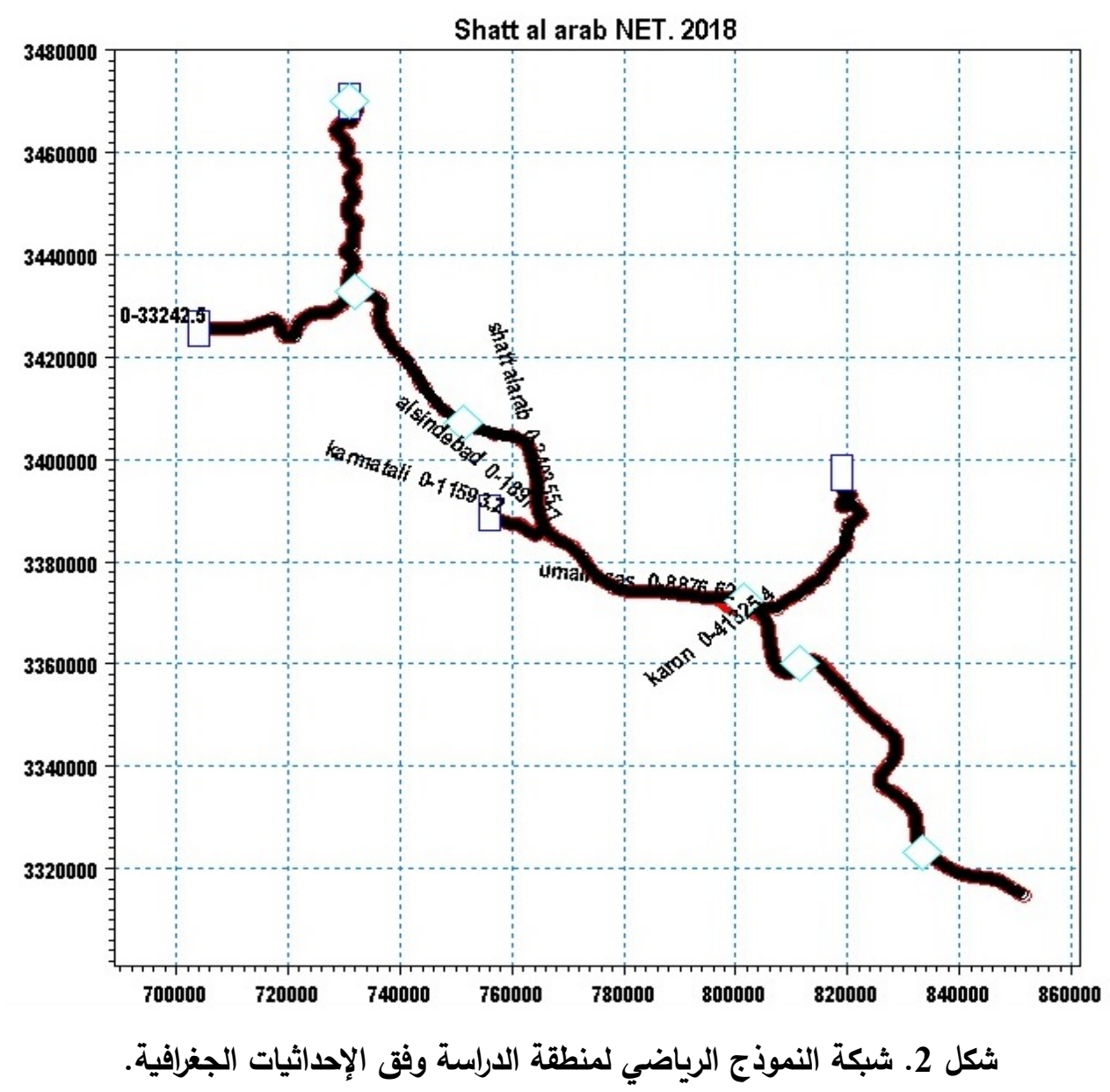

كبيرة في الأعماق على طول مسار النهر، فقد سجل

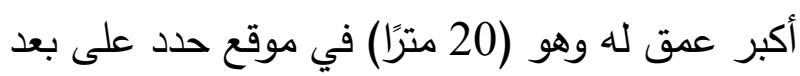
(120,000 كم) من أعلى النهر ، كما أن هنالك أعماق تصل إلى (18 مترًا)، في نقطة تبعد (117,000 كم)، إلا أن أقل عمق تم تسجيله هو (6 أمتار) في الموقع

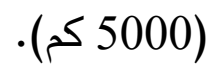

حيث يعتبر قاع النهر من الأشكال المعقدة في انحدار القاع، والتي تعمل على إعاقة التيارات الداخلة والنهايات كونه ليس له انحدار ثابت على طول النهر .
شكل 3 يوضح المقطع الطولي لشط العرب وفقاً

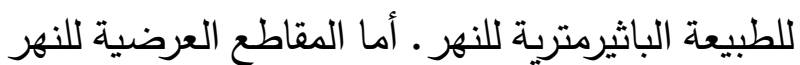
على طول (290 كم) فهي في كثير من الأماكن غير موجودة في الثكل حيث يبدو أن معدل عرضها (400 منال

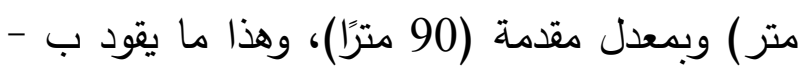
الاتحاد - عبر نموذج رياضي تحليلي ذي بعد واحد

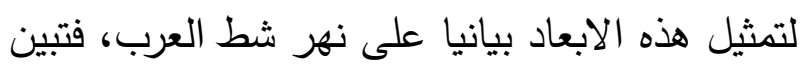

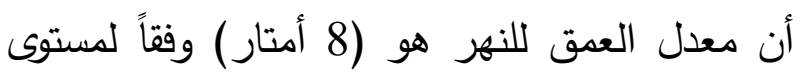
سطح البحر، إلا أن ما يلاحظ هو وقوع اختلافات 


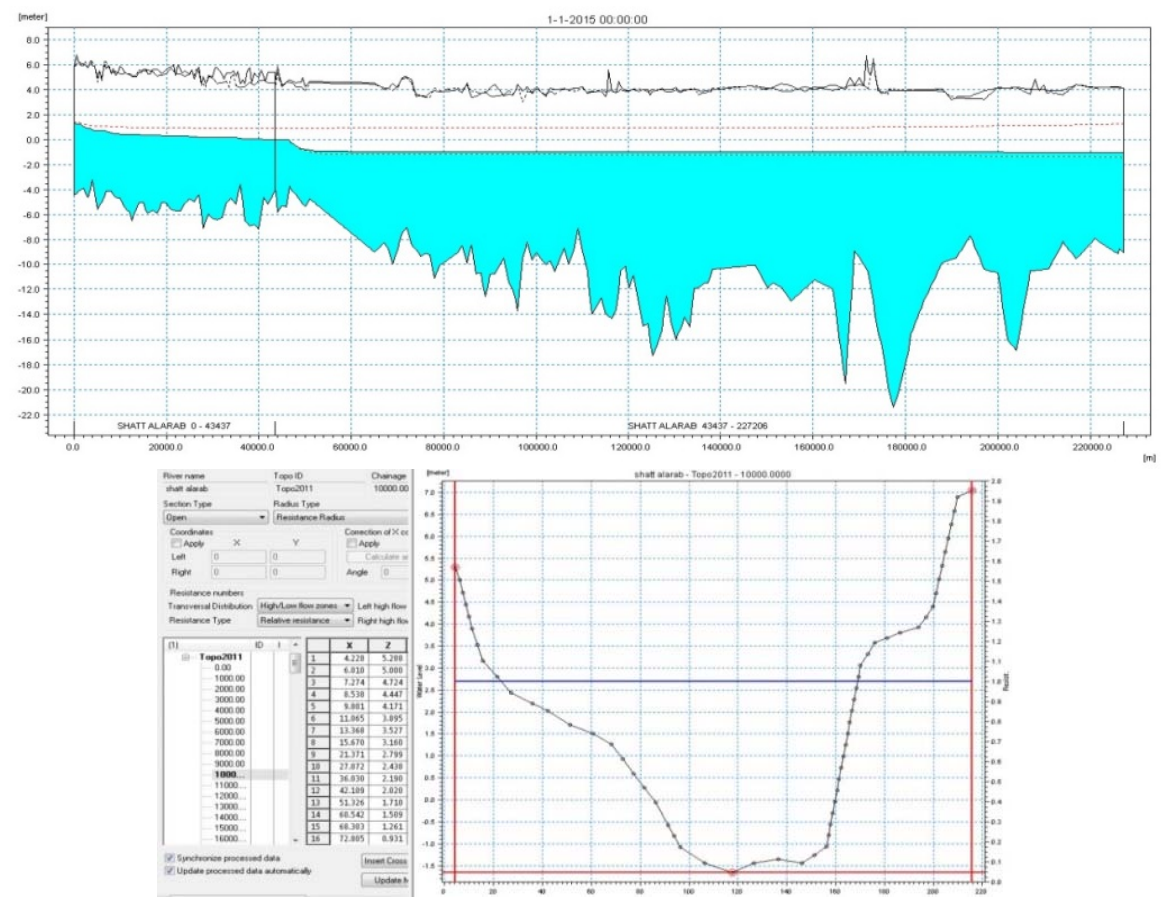

شكل 3. مقطع عرض طولي لقاع نهر شط العرب.

\section{ثانيًا: أسفل النهر (المقتربات السفلى)}

وهي عبارة عن متسلسلة زمنية لمدة ما (عدة سنوات) تبين مسار الانخفاضات والارتفاعات لمستوى سطح المياه عند (رأس البيشة) أسفل النهر الناتجة عن تأثير ظاهرة المد والجزر في هذه المنطقة، حيث تتصل هذه الظاهرة بالخليج العربي عبر خليج عمان عن طريق مضيق هرمز (شكل 4). فقد تم إدخال المتغيرين الرئيسيين وهما الملوحة في أول نقطة بالموديل بمقدار (1,5غم/ لتر) والملوحة في المقطع الأخير (في نهاية المنخفض) وهي (38)

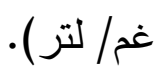

Boundary Conditions 1-3 الشروط الحدية اعتُمدت - في هذه الدراسة - اختبارات الثروط الحدية للدورات وفقاً للبيانات المتوفرة التي بني عليها هذا البحث حيث تتطلب مثل هذه الموديلات بعض المتغيرات في الحدود المفتوحة للنهر والمقتربات العليا والسفلى.

$$
\text { أوله: أعلى النهر (المقتربات العليا) }
$$

وقع الاختيار - جغرافيا- لتحديد عينة البحث محل الأشتغال على تصريف المياه العذبة القادمة من نهر دجلة من خلال (ناظم الكسارة) التي تعد مصدرًا رئيساً في الآونة الاخيرة لتجهيز نهر شط العرب بالمياه العذبة، حيث تم اعتماد القيمة (50 م مثر). 


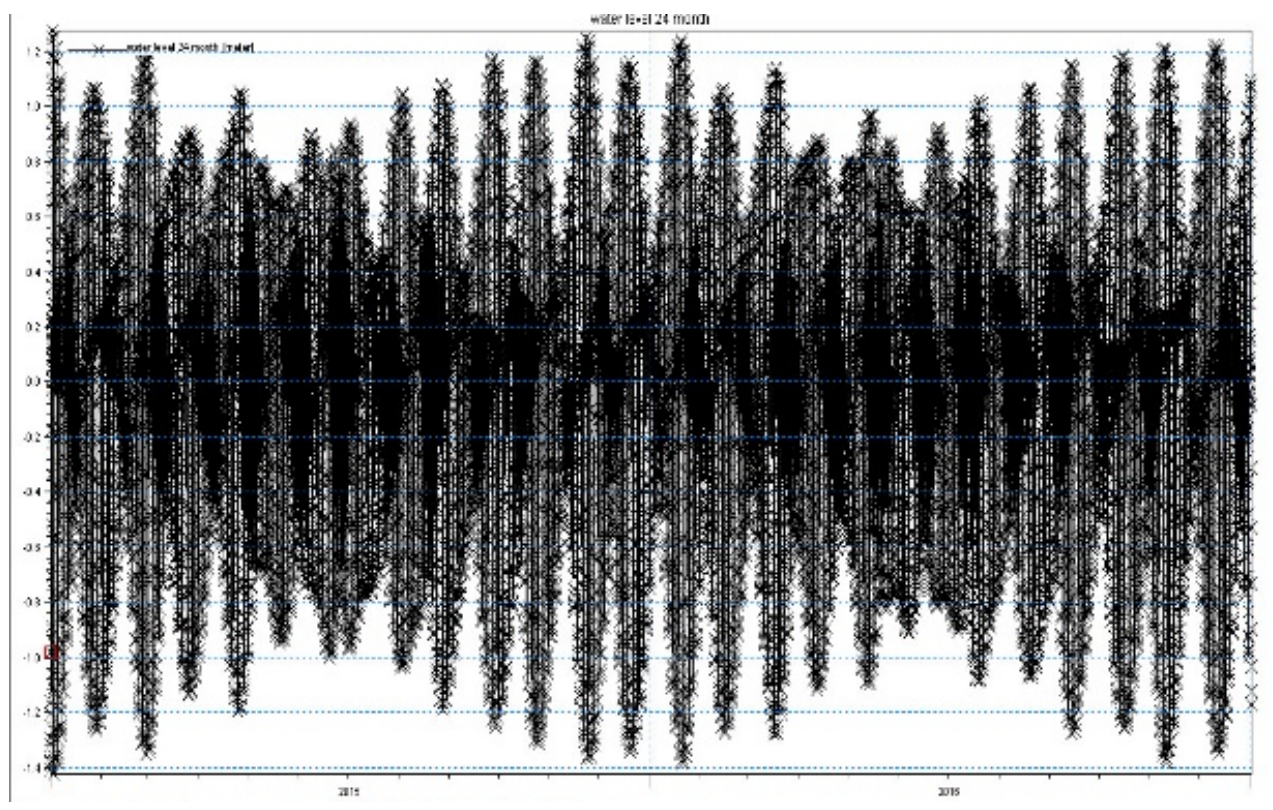

شكل 4. سلسلة زمنية لارتفاعات المياه البحرية (المد والجزر) عند ملتقى الخليج العربي-الفاو.

بعد اعتماد النموذج الهايدروديناميكي للنهر والذي تم استتاجه من محاكاة البرنامج للمتغيرين

الرئيسيين وهما الملوحة في أول نقطة وتقدر غم/لتز) والملوحة في المقطع الأخير (في نهاية المنخفض) والتي قدرت بـ (38غم/ لتر)، أما تأثير العامل الثاني وهو ظاهرة المد والجزر، والتي مثلت بالمتغيرات اليومية لمناسيب المياه في المقطع الاخير للنهر ولمدة سنة كاملة، فيبين شكل 5 مقدار تطابق النتائج الرياضية مع القيم المقاسة للتصريف ولدورة مدية كاملة (13 ساعة) ليوم 20/ 9/ 2017م في محطة القياس المعتمدة (السيبة).
4- المعايرة والتحقيق للنموذج

تمت عملية المعايرة من خلال رفع بعض قيم أعماق الأنهار لحركة المياه في الأسفل، وخاصة لمانه التشوهات في الأعماق لمقاطع محددة واقعة بين مقطعين متشابهين في العمل. ويتضح ذلك من خلال:

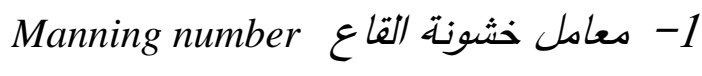
إذ أجريت معايرة الأنموذج الرياضي على قيمة تتراوح بين (0.025 - 0.03) كونها قيمة مناسبة

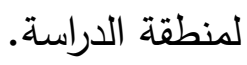
2- تصاريف المياه Discharge تمت مقارنة التصريف الحقيقي للنهر في منطقة البصرة مع قيمة التصريف الناتج عن الموديل، وقد تمت مطابقتهما لكمال عمل الموديل بالدقة المطلوبة. 


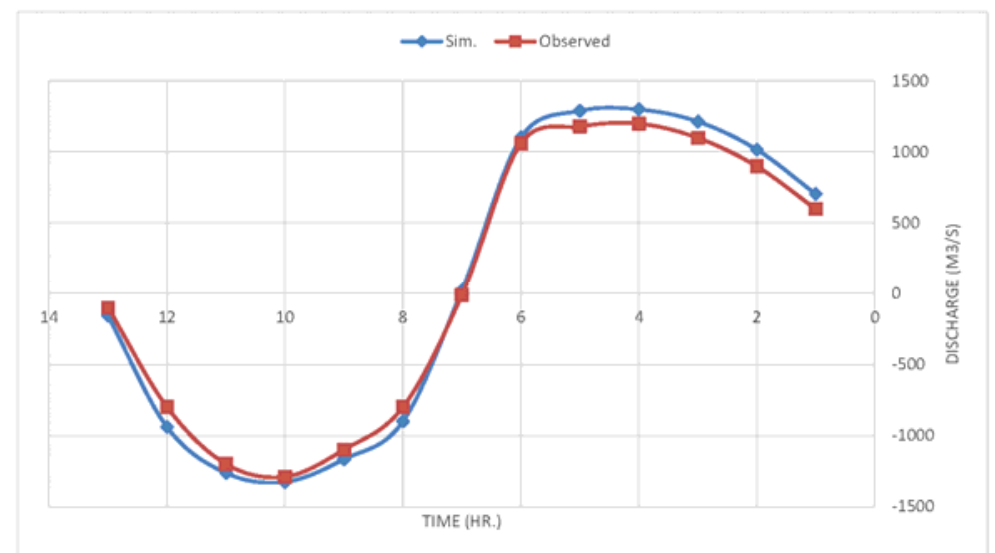

شكل 5. تطابق نتائج المحاكاة والقياسات الميدانية للتصريف لمنطقة السيبة في الطور الفيضي خلال دورة مدية كاملة ليوم .2017/9/20

أما في حال التصريف (50 مم/ ث) وبعد تثغيل البرنامج لمدة (3 أشهر ) أظهرت نتائج المحاكاة

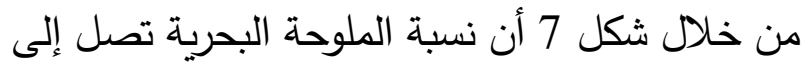
(14 غم/لتر) في نقطة (20 كم) أي في موقع يتجاوز منطقة (الدورة) أعلى النهر بأن ذلك من خلال الطور

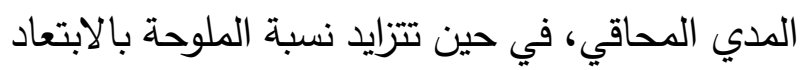
إلى مسافة أكبر تقدر (25 كم) في حال الطور الفيضي (spring plase). يظهر شكل 8 نتائج محاكاة السيناريو الأخير الذي

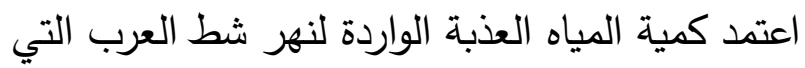
تقدر بـ (10 م³/ث) وبعد تشغيل البرنامج لأطول فترة

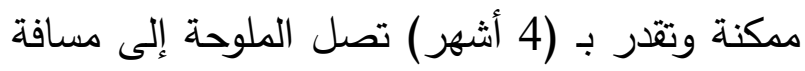
(120 كم تقريبًا) في الطور المحاقي وإلى مسافة (130 كم تقريبًا) في الطور الفيضي.

$$
\text { 3- النتائج والمحاكاة }
$$

بعد تثغيل البرنامج لفترات زمنية متعددة بينت النتائج

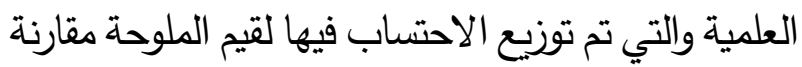
بالمسافة على طول النهر شط العرب في عدة أماكن مختارة (مثل: الفاو، والسيبة، والبصرة) كالتالي: 1) الاطلاع على النتائج بشكل تفصيلي يومي أو شهري (دورة مدية كاملة). 2)معرفة كيفية تصريف الأملاح وفقاً لقيم التصاريف المدخلة إلى النموذج. 3) إدرالك أية قيمة تصريفية للنهر تتوغل الملوحة إلى المحطات أعلاه (الفاو، والسيبة، والبصرة) وفقاً للفترة الزمنية المناسبة لكل تصريف. حيث أظهرت النتائج (كما في شكل 6) أن قيم تصريف المياة العذبة في أعالي منطقة الدراسة تصل

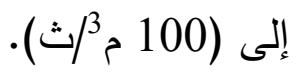



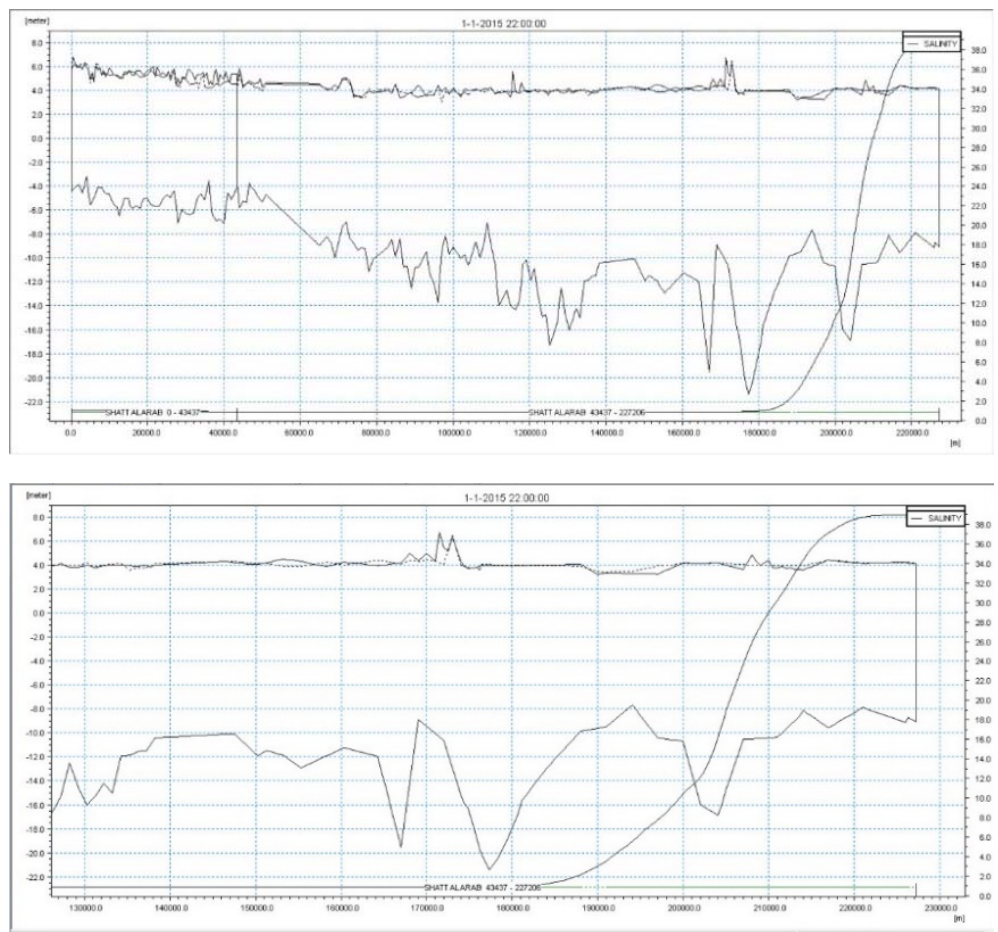

شكل 6. محاكاة التصريف للمياه العذبة الواردة لنهر شط العرب.
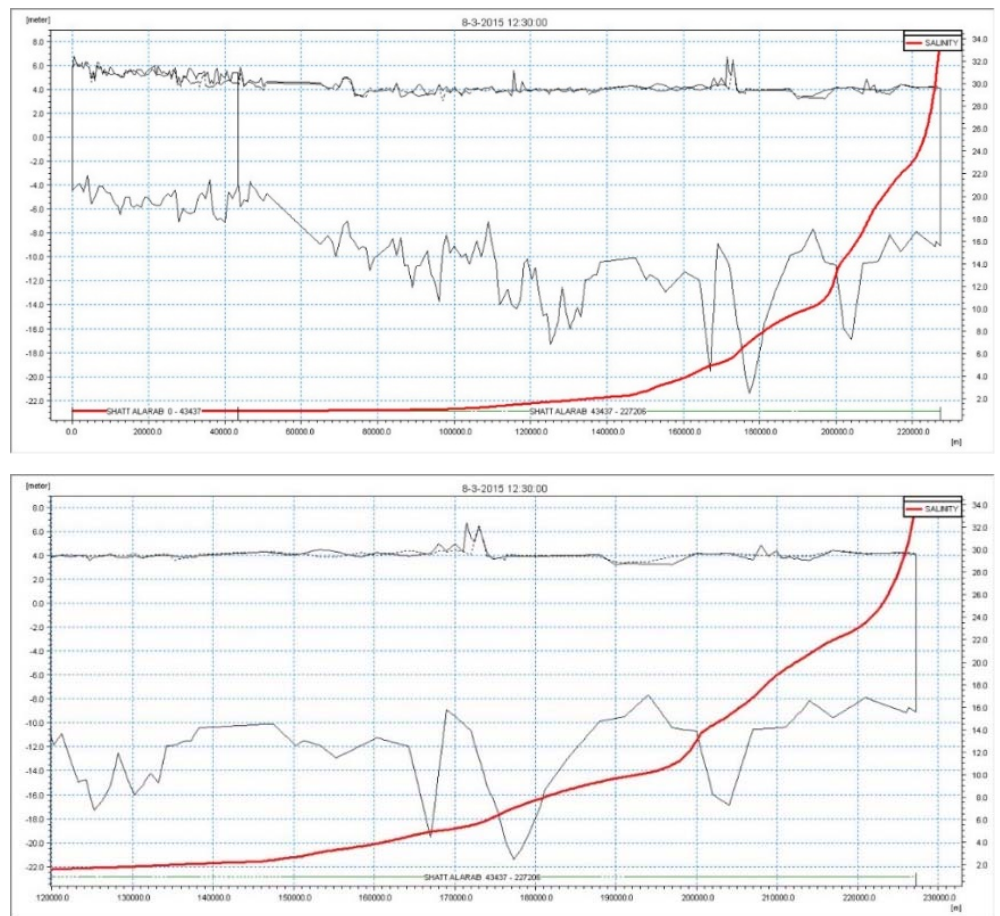

شكل 7. محاكاة التصريف للمياه العذبة الواردة لنهر شط العرب بمقدار 50 مم ث. 


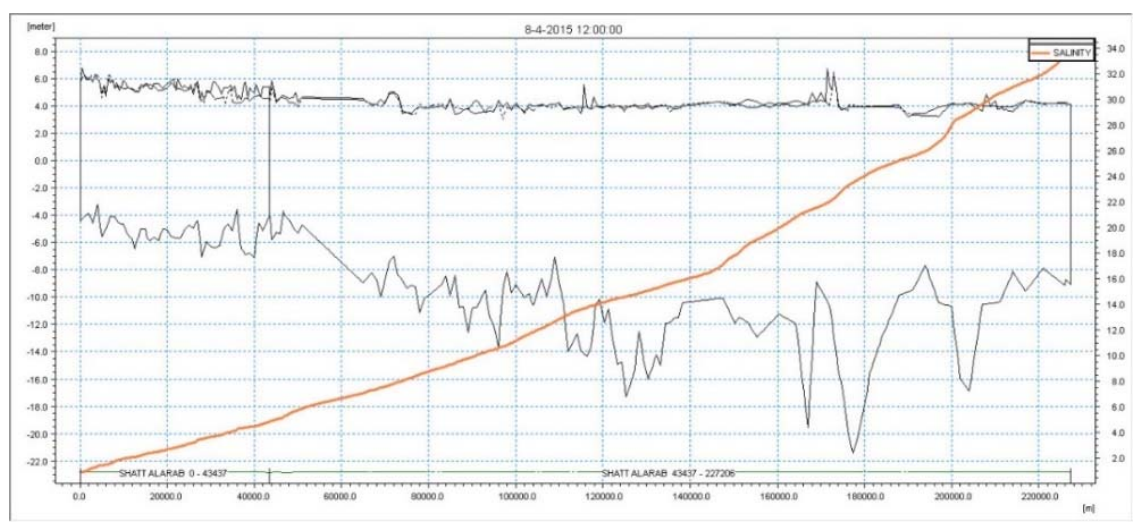

شكل 8. محاكاة التصريف للمياه العذبة الواردة لنهر شط العرب بمقدار 10 مم ث.

حيث وصلت الأملاح البحرية إلى مسافة تقدر بـ (20) كم) أعلى النهر خلال فترة زمنية تقدر بـ (5 أيام) عندما يُجَهَّز النهر بتصريف يصل إلى (100 مم ث)، ووصلت إلى مسافة (120 كم) عند مدينة البصرة بعد (120 يوما) بسبب انخفاض التصاريف دون (10) م³/ث)، وبقيمة ملحية كانت بحدود (12غم/ لتر). أما عند التصريف الحالي والذي يقدر بـ (50 م³/ ث) فتصل الأملاح البحرية إلى (45 كم) وتزداد تلك القيمة لتصل إلى (6 غم/ لتر) عند محطة السيبة بعد قضاء

$$
\text { شدة (90 يومًا). }
$$

لا يسعنا إلا أن نقدم شكرنا وتقديرنا لـ(DANEDA)

MIKE ووزارة الخارجية الدنيماركية لمنحها حزمة برامج لمركز علوم البحار-جامعة البصرة، والثكر موصول أيضا إلى مديرية الموارد المائية في البصرة، وكادر مركز علوم البحار، على التعاون والمساعدة بالحصول على البيانات المطلوبة لإنجاز البحث.

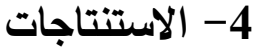

• يعد برنامج Mike11 من الوسائل التكنولوجية الرائدة التي تعطي تصورا علميا دقيقاً وواضحاً حول مديات ونسب توغل الأملاح والذي تتصح الدراسة بالتعويل عليه، واعتماده كقاعدة بيانات تدرج فيه التغيرات المستقبلية للتنبؤ بمخرجاتها بالسرعة الممكنة؛ لاتخاذ القرارات والحلول المناسبة.

• إن Mike11 من البرامجيات الأكثر فرعية في استخدامها للأنهار والقنوات المفتوحة من بقية النماذج الاخرى كونه يقدم بيانات دقيقة للمحاكاة HD. Hydrodynamics الهيدروليكية والهيدرولوجية ويشمل إضافات علمية واعدة منها عملية التوغل الملحي في الأنهار والمصبات. • اعتمدت الدراسة مقدار مسافة توغل الأملاح البحرية من الخليج العربي إلى داخل شط العرب وفقاً لسياق الزمن وكمية التصريف القادمة من أعلى النهر ، 


\section{ثانيا: المراجع الأجنبية}

سامر عدنان الطائي وآخرون

Abdullah, Sadiq Salim (2002). Analysis of tide wave in Shatt Al Arab Estuary, Marina Mesopotamica, 17(2): 305315.

Al-Taei, S. A., Abdulla, S. S. and Lafta, A. A. (2014). Longitudinal intrusion pattern of salinity in Shatt Al-Arab estuary and reasons. JKAU: Mar. Sci., 25(2): 205-221.

Cai, H., Savenije H.G., Zuo S., Jiang C. and Chua, V. P. (2015). A predictive model for salt intrusion in estuaries applied to the Yangtze estuary. Journal of Hydrology, 529, Part 3:1336-1349.

Center of Studies and Engineering Designs (CSED), Ministry of Water Resources, Republic of Iraq; Medingegneria; Studio Galli Ingegneria and EI Concorde Construction (2012). Shatt Al-Arab Irrigation Project, Technical Report, Vol. IX, Studies on salinity problems. p:255.

Danish Hydraulic Institute, DHI (2007). Mike1 1a modeling system for river channels. Short introduction tutorial. http;//www.dhigroup.com.

General Establishment for Studies and Designs (GESD) Ministry of Irrigation, Republic of Iraq; Polservice and Hydroproject (1981). Shatt Al-Arab Project, Feasibility Report, Volume IX , p:316, (Unpublished) .

Gong, W. and Shen, J. (2011). The response of salt intrusion to change in river discharge and tidal mixing during the dry season in the Modaomen Estuary, China. Continental Shelf Research, 31:769-788.

Grass, S. and Saveniji, H.H. (2008). Salt intrusion in the Pungue Estuary, Mozambique: effect of sand banks as natural temporary intrusion barrier. Hydrology and Earth System Science Disscussions. 5:2523- 2542.

Leblond, P.H. (1978). On tidal propagation in shallow rivers. J. Geophysical Res. 38:4717- 4721.

Vongvisessomjai, S. and Catanantavet, P. (2006). Analytical model of interaction of tide and river flow in. Songklanakarin. J. Sci .Technol., 28(6):1149-1160 .

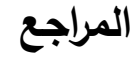

أولا: المراجع العزبية

مركز علوم البحار، قسم الفيزياء البحرية (2014). الدد

والجزر ومصادر الأملاح. تقرير علمي منشورات مركز البحريه

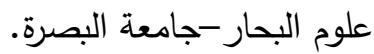

مركز علوم البحار، مكتب الاستثارات البحرية (1998).

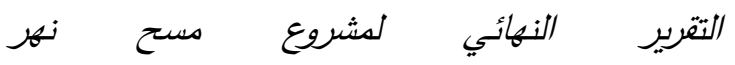

دجله بين العمارة والبصرة من ناظم الكسارة إلى الفاو.

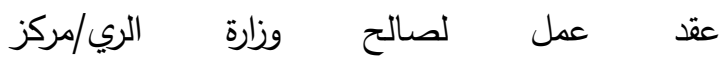

الفرات للدراسات وتصاميم مشاريع الري (دراسة غير

منشورة).

وزارة الموارد المائية، مديرية الموارد المائية في البصرة

(2016). القياسات الحقلية لنهر شط العرب ونهر دجلة العهاتيه

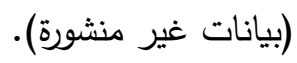

عبد الله، صادق سالم (1990). دراسة في الحمولة النهرية لثط

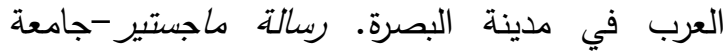

البصرة، 115 صفحة، العراق، البصرة .

عبدالله، صادق سالم (2014). ظاهرة المد والجزر في شط العرب، جنوب العراق. مجلة الذليج العربي. 42 (3-4). 


\title{
Simulation of the Salinity Intrusion into the Shatt Al - Arab River, South of Iraq
}

\author{
Samer Adnan Al Taei ${ }^{1}$, Sadiq Salim Abdullah ${ }^{1}$ and Ayad Al-Mahdi ${ }^{2}$ \\ ${ }^{1}$ Marine Physics Dep., Marine Science Centre and ${ }^{2}$ Geography Dep., College of Education for \\ Women, University of Basrah, Iraq \\ samer.adnan74@gmail.com
}

\begin{abstract}
MIKE 11 program was used to simulate the intrusion of the salinity from the Arabian Gulf to the Shatt al-Arab River, due to impact of the tidal phenomenon in the region. The simulation program has been prepared for a period of six months and using many scenarios for different fresh water discharge as $10 \mathrm{~m}^{3} / \mathrm{sec}, 50 \mathrm{~m}^{3} / \mathrm{sec}$ and $100 \mathrm{~m}^{3} / \mathrm{sec}$, with account in consideration the only supplier of freshwater is the Tigris River and the Karun River is closed. The results showed that the salinity is transported from its source, the Arabian Gulf, to the center of the city of Basra after about four months with the value of discharge is $10 \mathrm{~m}^{3} / \mathrm{sec}$ and Salinity values are recorded $12 \mathrm{~g} / \mathrm{l}$ in this case. In the case of discharge of $50 \mathrm{~m}^{3} / \mathrm{sec}$, the salinity values range from 2-2.2 g/l at the center of Basrah and 7-6 g/l at the Saybah station, while When use the discharge of $100 \mathrm{~m}^{3} / \mathrm{sec}$, the salinity values will be to $4 \mathrm{~g} / \mathrm{l}$ at the FAO station in the ebb condition. It can be concluded that the intrusion of the salinity reach a longer distance in the Shatt al-Arab depends mainly on the tidal phase, especially spring phase and the little amount of freshwater discharge.
\end{abstract}

Keywords: Salts intrusion, Simulation, MIKE11, Fresh water drainage, Shatt Al-Arab estuary. 
